\title{
La interpretación morfológica de la tésera celtibérica K.0.6 y los derivados de temas en nasal en celtibérico
}

\author{
Carlos Jordán Cólera \\ Universidad de Zaragoza \\ cjordan@unizar.es
}

The morphological interpretation of the Celtiberian tessera K.0.6 and the nasal stem derivatives in Celtiberian language

\begin{abstract}
En este artículo se trata de explicar desde el punto de vista morfológico lo que realmente se lee en la tésera celtibérica K.0.6, sin tener que suponer un lapsus del grabador. A partir de esas explicaciones se realizan unas consideraciones sobre los derivados de temas en nasal en celtibérico.
\end{abstract}

Palabras clave: epigrafía celtibérica; lengua celtibérica; derivación; temas en nasal.
This paper discusses from morphological point of view the correct reading of the Celtiberian Tesserae K.0.6, refuting the previous explanations based of an 'engraver's error'. The alternative reading also allows the author to draw some conclusions on the adjectives derived from nasal stems in the Celtiberian language.

Key words: Celtiberian epigraphy; Celtiberian language; derivation; nasal stems.

En el número 51 de la revista Emerita, Tovar dio a conocer «Una nueva pequeña tésera celtibérica». Corría el año 1983. La pieza, según indica el autor, se perdió en el comercio de antigüedades y tuvo que trabajar sobre las fotografías en color, calcos y dibujos que había efectuado el Dr. P. Rubio Requena.

Era una pieza de bronce en forma de jabalí, partida por la mitad longitudinalmente, con unas medidas máximas de $5,2 \mathrm{~cm}$ de largo y 2,6 de alto. En la cara plana se observaban seis signos incisos, pertenecientes al signario paleohispánico oriental que Tovar leyó como atulịkum. En el comentario paleográfico decía (p. 2): «Únicamente no es normal la $i$, que es como una $n$, con falta del medial de los rasgos superiores». Desde el punto de vista lingüístico, analizaba atulịkum como el «genitivo de plural de una formación adjetival sobre el nombre personal Atulus (posible también Atullus, o acaso 
Adul(l)us)». Los paralelos que ofrecía eran en el Bronce de Ascoli, Atullo Tautindals $f$., dentro de los suconsenses, que declina como un tema en nasal (Atullo, -onis); Adulus (CIL XII 1403, Vasio); Atullus (DAG 202, Narbonense); Atula (CIL V 4117, Cremona, como nombre de una mujer en una inscripción cristiana); Atuli f. (CIL III 11556, Carintia, Austria); Atu[llius] (CIL XIII 6085, Rheinzabern, Alemania); y Atull de una moneda del sur de la Germanica céltica. El antropónimo no estaba documentado en la Península Ibérica, hasta que Mesa 1998 presentó un grafito sobre cerámica hallado en el Foro de Caesaraugusta en el que leía Atullus. Comentaba este autor que la pieza está rota «justo en el arranque de la A que inicia el grafito» (p. 198), con lo que se podía pensar que no estaba completo el antropónimo y leer Satullus, Statullus o Catullus. En la página siguiente (p. 199), indicaba, sin embargo, que «una autopsia detenida ... no ofrece el menor indicio de que algún tipo de grafía precediera a la vocal - $a-\gg$. La lectura definitiva del autor era Atullus, aunque insistimos que la pieza está rota.

La lectura e interpretación de Tovar han sido las que se han manejado habitualmente, siempre que se ha hecho uso de la pieza. Es más, la lectura se «corrigió» muy pronto como atulikum, sin siquiera hacer referencia a ese punto conflictivo de lectura, que indica el editor. Valgan a modo de ejemplo, De Hoz 1986, pp. 70-71, con la lectura ya atulikum; Untermann 1990, pp. 357-358, n. ${ }^{\circ}$ 9; $M L H$ IV (K.0.6), pp. 546-547; Curchin 1994, p. 94; Villar 1995, p. 95; Beltrán 2001; Almagro-Gorbea 2003 (CP-11); Wodtko 2000, s. u. atulikum; Almagro-Gorbea et al. 2004, pp. 308-309; Jordán 2003; Ballester 2004, p. 279; Jordán 2004a, p. 314; 2004b; 2004c, pp. 259-260; Balbín 2006, p. 180; Jordán 2007, pp. 115-116.

Afortunadamente, la pieza reapareció. La Real Academia de la Historia adquirió el 15 de noviembre de 2002 la Colección de D. Josep Pellicer i Bru de veinticuatro téseras prerromanas. Entre ellas se encuentra la que aquí tratamos, que fue catalogada con el número de inventario 2002/25/1 (AlmagroGorbea 2003, pp. 380-381 y 392, donde se recoge con la referencia CP-11). Realizamos autopsia en dos ocasiones, los días 21 y 22 de junio de 2006 y el 23 y 26 de septiembre de $2008^{1}$. Las dos últimas tuvieron lugar dentro del Proyecto de Investigación «Identidades y diversidad cultural en la historia antigua: bilingüismo y cultura epigráfica» (HUM2006-13424-C04-02/FIL),

\footnotetext{
${ }^{1}$ Queremos agradecer desde estas líneas a los doctores M. Almagro-Gorbea y J. Maier las facilidades dispensadas al realizar estas visitas.
} 
junto con los doctores F. Beltrán e I. Simón, en el que intentamos llevar a cabo la revisión del mayor número de piezas consideradas téseras y cuyos resultados presentamos de forma preliminar en el Coloquio de Lenguas y Culturas Prerromanas celebrado en Lisboa en febrero de 2009 (Beltrán, Jordán y Simón 2009). La lectura que allí ofrecemos es: atulnkum (!).

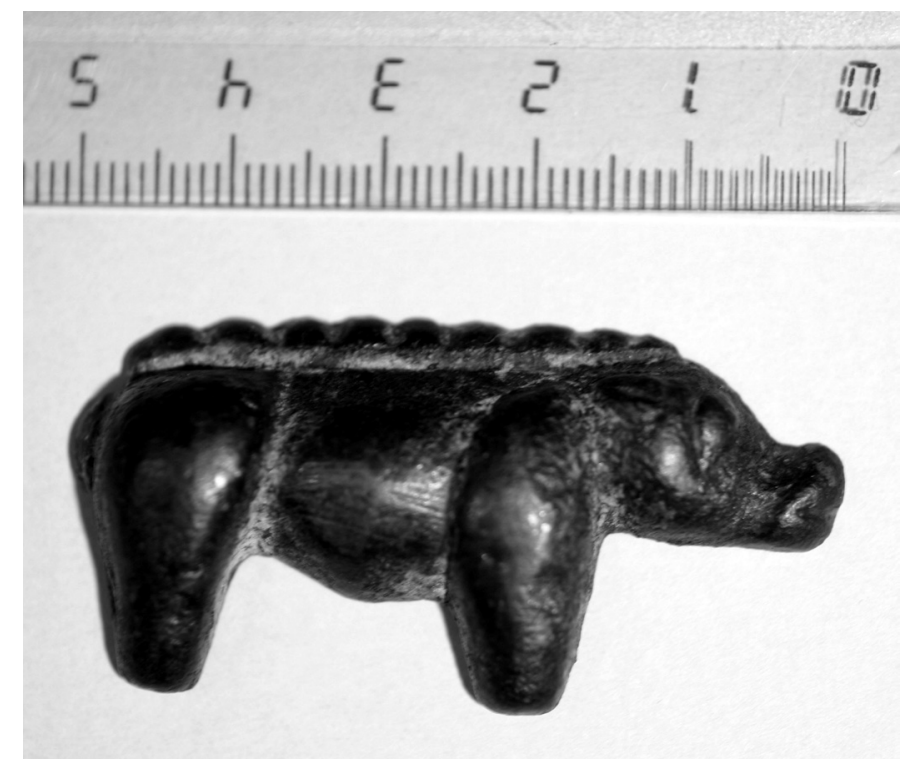

K.0.6 Cara A (Foto: F. Beltrán)

La razón es clara. El cuarto signo es una $n$ oriental y no hay trazas de restos, de dudas o de intentos de haber trazado una $i$. El signo de exclamación recoge, en definitiva, la sorpresa de leer algo que no es lo esperado. Hay que aclarar que lo esperado es lo que Tovar dejó sentado en su lectura, que a su vez se ajusta (no al revés) a su intepretación morfológica: el nombre familiar en cuestión es un adjetivo derivado con el sufijo -iko- de un antropónimo, que es un tema en $-o$, en casi todos los ejemplos aducidos. Decimos en casi todos, porque hay uno que no lo es: Atullo del Bronce de Ascoli. Tovar lo declinó como nombre en nasal, Atullo, -onis, y la verdad es que no hay nada que se oponga a ello. Siempre se prodrá argüir que bastantes de los nombres que aparecen en ese documento no están adaptados a patrones morfológicos 
latinos, pero también es cierto que los hay que sí que lo están o al menos lo parece (sobre esta cuestión uid. Jordán 2008).
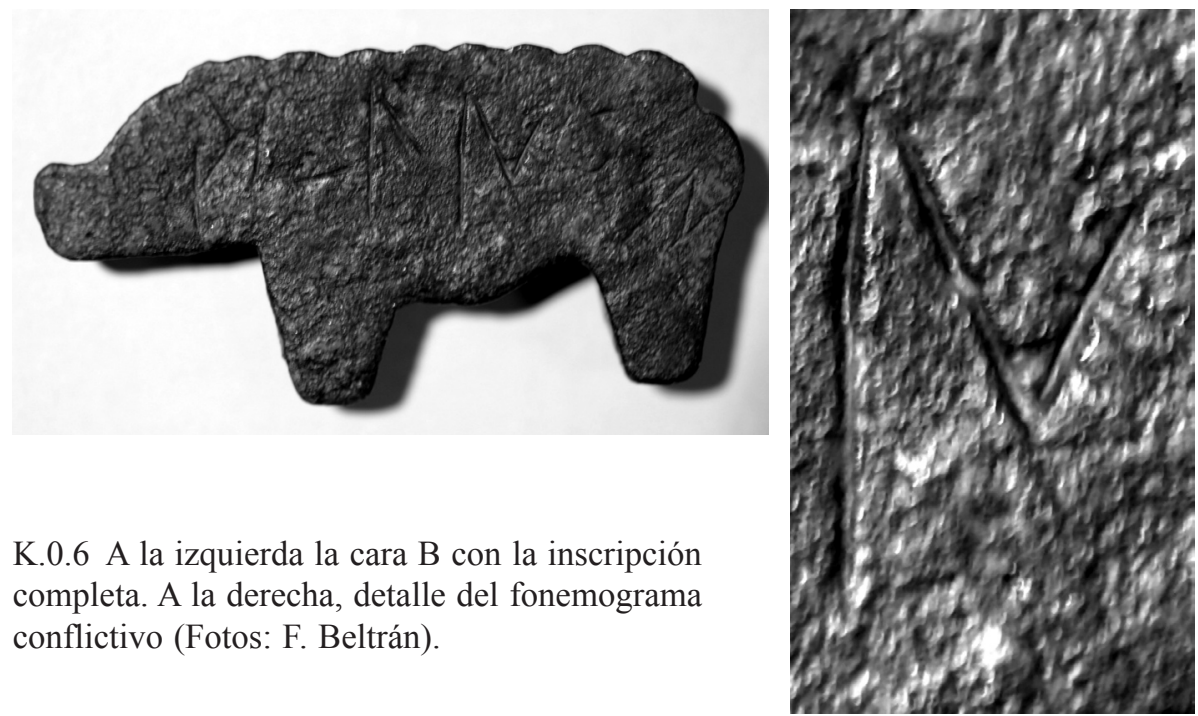

K.0.6 A la izquierda la cara B con la inscripción completa. A la derecha, detalle del fonemograma conflictivo (Fotos: F. Beltrán).

La consideración de que Atullo sea un tema en nasal y de que el lapsus del grabador sea precisamente entre una vocal y una nasal nos obliga a intentar seguir el sentido contrario al que se ha venido tomando hasta ahora: aceptar la lectura e intentar encontrarle explicación morfológica. Vemos dos posibilidades:

A) Considerar que la lectura de atulnkum esté reflejando una secuencia fónica aproximada [atulnkūm] y que el idiónimo del que hay que partir es el tema en nasal *atulō(n). La explicación queda como una formación con sufijo - $k o$ - sobre el tema con el sufijo individualizador en nasal -on- en grado cero, *atul-n-ko-. Esto supone aceptar un patrón apofónico [tema fuerte con sufijo *-on / tema débil con sufijo en grado cero *-n-].

La posibilidad, remota a nuestro juicio, de derivación a partir del sufijo posesivo ${ }^{*} h_{3}$ on- exigiría suponer algunos fenómenos fonéticos que, aunque viables, no podemos confirmar de momento, *atul- $h_{3} n-k o->* a t u l-a n-k o->$ *atulnko-: con síncopa de la vocal procedente de la vocalización de la laringal por las razones que fuesen o incluso de la laringal originaria, pasando por 
alto que el sufijo no se añade a un tema en -o, según lo más esperado, pues si fuese así entonces la síncopa sería la de una vocal larga.

La formación con el sufijo -on- en grado cero ya ha sido propuesta para otros casos de genónimos. Así, Hamp 1989, p. 188, propuso que abulokum $<* a b l n o k o m$, donde la líquida vocalizaría como -ul-, ante vocal posterior de la sílaba siguiente (y lo mismo servía para el nominativo del idiónimo abulu $<* a b l \bar{o}$, genitivo abulos $<* a b l n-o s$, y las formaciones en epigrafía latina con Ablo-); para abiliko[ (K.8.1) proponía partir de *ablniko, de una base *abl-n-, con vocalización -il- por la vocal de la serie anterior de la sílaba siguiente (de igual forma en las formas en epigrafía latina Abilius, Abilicorum y suponemos que también para abilikum [K.1.3, IV-7], forma que Hamp no podía conocer cuando escribió su artículo).

Wodtko 2000, s. u., admitía esta posibilidad para:

1. abulokum <*abul-n-okum, el tema en nasal, aunque no puede rechazar partir de un tema en -o*abul-o-s. No está testimoniado de momento ese tema en $-O$.

2. statulikum (K.1.6, B-6): *statul-n-ikum > *statullikum, aunque admite que no hay que desechar del todo partir de *statulos, con el correspondiente sufijo. Aquí sí que existe Statullus en el sur de la Lusitania (D’Encarnação en IRCP 189) y en la Bética (CIL II 2005), cuya homofonía con el antropónimo celtibérico tiene que ser casual a juicio de Untermann (BB III, p. 154).

3. oilaunikos (A.56) <*oilaun-n-ikos, cf. nominativo oilaunu, ablativo oilaunez $<*$ oilaun-n-ed. Aquí la autora no dice nada sobre un hipotético tema en -o $i^{*}$ oilaunos? Este adjetivo no es un genónimo como los dos anteriores.

La diferencia entre estas tres formaciones y la que estamos aquí tratando está clara. En las primeras la aparición de una vocal tras la nasal deja una secuencia *-VlnV-, que, según se admite de manera general, debe evolucionar a $-V l l V$-, tal y como parece confirmar el genitivo abulos $<*$ abulnos del nominativo abulu $<* a b u l o ̄(n)$ y como, según estamos considerando ahora, debió de llegar a suceder en el genitivo de *atulū que acabaría siendo *atulos procedente de una etapa anterior *atul-n-os. Esto significa que el correspondiente adjetivo *atul-n-kos debió conformarse antes de que se produjese la asimilación, $-l n->-l l-$, y que conservó la nasal porque no se encontraba en 
el mismo entorno fonético que la forma del genitivo nominal, con una silabación *a-tuln-kum.

La restricción gráfica del signario paleohispánico de no marcar las geminadas mediante la reduplicación gráfica no permite confirmar al cien por cien la derivación de abulokum, abiliko[/abilikum y statulikum, a partir de los correspondientes temas en nasal, y se prefiere la alternativa de partir de la formación temática correspondiente, línea de explicación marcada por Villar 1995, p. 144, y Untermann en $M L H$ IV 427. Ésta es por la que opta Rubio 2001, pp. 582-584, para explicar, las siguientes formas, a las que etiqueta como «con deleción de $-\bar{u}(n) »$ :

— abulokum (K.16.1) y abilikum (K.1.3, IV-7) a partir de abulu (K.1.1, A-11, etc.).

- akikum (K.1.3, II-12, IV-29), Acciq(um) a partir de Acco, - $a$ (para localizar las referencias en epígrafes latinos puede consultarse Abascal 1994 y Vallejo 2005).

— alikum (K.1.3, III-30, etc.) a partir de alu, Allo, - $a$.

- amikum (K.0.11) a partir de amu, -a, Ammo, - $a$.

- atokum (K.1.3, passim) y atiko (K.0.7), de atu, Atto, -a, At(t)us.

- austikum (K.1.3, I-20, II-14) de *austu?, a partir del cual tenemos austunikum (K.1.3, III-5, IV-31).

- beskokum (K.1.3, III-27) de besku (K.5.1).

- kariko (K.6.1) y Cariqo(m) de Caro, -us, - a.

- elokum (K.1.3, III-28) de elu (K.1.3, II-5, III-39), Elae.

- Letondiq(um) de letontu (K.0.8, -1), (K.1, B-1), etc.

— litokum (K.1.1, B-1) y, con dudas, letikum (K.1.1, B-8) de litu (K.1.3, I-57, IV-8).

- oilaunikos (A.56) de oilaunu (A.56).

— statulikum (K.1.6, B-6) de statulu (K.1.3, I-3).

- Stennoco, que es una lectura errónea en lugar de Stennico, como se verá a continuación, de stenu (K.1.3, III, 6), stena (K.1.3, I-16, III-13, III-19), Sten(n)a.

— tetokum (K.1.3, I-31, II-20, II-38) de tetu (K.1.3, III-18).

- Turiasica (K.27.1) de turiazu (A.51).

Puede observarse que además de las ya referidas abulokum, abilikum y statulikum, admiten un análisis de un derivado a partir del tema en nasal: 
1. alikum (K.1.3, III-30, etc.) <*al-n-ikōm, de alu (K.1.3, I-43). Hasta la aparición de la obra de J. M. ${ }^{a}$ Vallejo (2005, pp. 114-166) sólo hay un caso en la Península Ibérica de Allus, tema en -o, y es dudoso (CIL II 1020, Badajoz). El resto es tema en nasal, con un testimonio allu y otro [Al] lonis (AE 1988, 781, Clunia, Burgos) en Celtiberia, un All[on]is (HEp 4, 538) en Monte Cildá (Palencia), zona turmoga, y otro All[on]is en zona vaccea (IRPP 16, Albertos 1972, p. 8). Hay más ejemplos fuera de esos territorios.

2. elokum (K.1.3, III-28) < *el-n-okōm, de elu (K.1.3, II-5, etc.). Tampoco se ha encontrado hasta el momento el correspondiente tema en - $o$, *elos, *Elus. Sí en cambio el tema femenino en -a, Ela (AE 1987, 618b, Burgo de Osma, Soria: Octaua Elae).

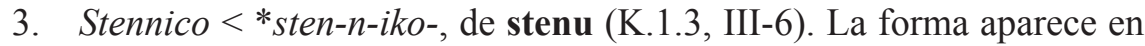
la inscripción Medittus . Stenni/co(n) . Lo[i...] . F(ilius) an(norum) . LXXV. / H(ic) . S(itus) . E(st) (Heras Fernández 1993). Parece, por lo tanto un genitivo del plural. De nuevo aquí, de momento, sólo tenemos testimoniado el tema en nasal para el masculino y el tema en - $a$ para el femenino, stena (K.1.3, I-16, III-13, III-19), Stena (HEp 2, 123, Coruña del Conde, Burgos) y Stenna (EEp 9, 319, Albarracín, Teruel).

4. mailikum (K.1.3, I-25, -30, II-23, IV-20). Este genónimo no está recogido en el cuadro. Lo consideramos porque presenta un panorama bastante curioso. Está testimoniado el tema en nasal Mailo/Maelo y su respectivo femenino Maila/Maela en la Lusitania. En la Celtiberia todavía no se ha hallado, que sepamos. Sí que aparece mailikinokum (K.1.3, III-52) y mailikinokum (K.1.3, III-60). Las parejas aparecidas en la Lusitania pueden permitir pensar en un estado igual en la Celtiberia, de modo que mailikum $<*$ mail-n-ikōm.

En ellas cuatro, todavía no se han encontrado las teóricas bases temáticas de las que hacerlas derivar, argumento por otra parte de poco valor, $\mathrm{y}$, además, se dan las condiciones fonéticas para llegar a ese resultado.

Lo que es cierto es que si la lectura fuese realmente atulikum también podría suponerse un punto de partida *atul-n-ikum $>$ *atullikum.

$B$ Considerar que atulnkum estuviese reflejando una secuencia fónica aproximada [atlūnkūm] y que el referente del que hay que partir es el tema en nasal nominativo *atlu $<*$ atlo $(n)$, genitivo *atlünos, en donde por exten- 
sión aparece el grado alargado del sufijo, al modo de melmu (K.1.1, B-2), melmunos (K.1.1, B-1).

A día de hoy no hemos encontrado el antropónimo *atlū, atlūnos testimoniado. En cuanto a formas relacionadas, podemos indicar que en CIL II 2387 (Iglesia de Santa Marinha de Ribeira de Pena, concelho de Villareal, Portugal) se dan dos lecturas de una inscripción cuya primera línea aparece como I/l/elus y como Atlus, y que en el aparato crítico se deja como . . . . us. Albertos 1977, p. 40, se debate entre una lectura Antelus, muy dudosa, y otra Atlus, también rara para la autora. D'Encarnação 1975, p. 127, parece aceptar Atlus. Abascal 1994, p. 289, en su entrada Atlus recoge [At]lus. Tal y como puede verse, es una lectura dudosa que no podemos utilizar en nuestra ayuda como argumento definitivo, pero tampoco hay que desecharla en tanto no se elimine fehacientemente.

Tampoco nos son de ayuda definitiva las lecturas Atlondi que se recogen en CIL II 76 Ourique (Ourique, Beja) y CIL II 4980, Garlitos (Badajoz). Para la segunda es clara la corrección Aplondi, al menos tal y como puede apreciarse en la fotografía que se ofrece en www2.uah.es:CILII7.0878.jpg. Para el testimonio de Ourique, cuyo paradero actual es desconocido, en la entrada del CIL se indica que Cenáculo daba la lectura Aplondi y es ésta la que recoge D'Encarnação en $I R C P$ 193, quien transcribe sus palabras: «Não há dúvidas nas letras senão na figura $\mathrm{P}$ que poderá ser I por ter a cabeça muy pequena e mal figurada». D'Encarnação apela también al testimonio de Palomar 1957, p. 37, y Albertos 1966, p. 29, que restituyen Aplondi apoyándose en los ejemplos de CIL II 4980, ya visto, y en CIL II 3082, donde no parece haber dudas en la lectura Aplondus. Como no podemos confirmar la lectura Atlondi, preferimos dejarla a un lado. En honor a la verdad, tampoco puede confirmarse la de Aplondi.

El último posible paralelo que hemos encontrado es Atlia, procedente de la necrópolis del Albarregas (Mérida, Badajoz). Ramírez y Gijón 1994, p. 153, prefieren esta lectura en lugar de la alternativa Atilia, en la que la $i$ que hay tras la dental habría quedado embebida en ella o no se habría indicado por descuido del lapicida. Esto les obliga a aceptar Atlia como cognomen y Specia como nomen, en contra de lo esperado y de lo que aparece en el resto del documento, excepto en otro lugar donde encuentran la misma estructura cognomen + nomen, Fausta Coelia. No es concluyente esta explicación, según nos indica el Dr. F. Beltrán, al cual agradecemos la observación. Tampoco queremos aprovechar un descuido del lapicida en un trabajo como el que aquí estamos presentando. 
Quizá el mejor paralelo podamos buscarlo en el ya indicado Atullo del Bronce de Ascoli. Planteamos para ello la posibilidad de que la forma que aparece en el documento latino sea la adaptación a esa lengua de un idiónimo indígena *Atlū(n). En este proceso estarían implicados:

1. El incómodo grupo o secuencia - $t l$-, que fue resuelto mediante la introducción de una vocal de apoyo $u$. Este fenómeno ya fue detectado por Correa 1983 a propósito de la leyenda monetal kaśtilo (A.97). Según este autor, el topónimo debía de ser aproximadamente [kastlo] tal y como parece indicar la forma Castlosaic CIL II 3294 y las referencias en textos griegos de

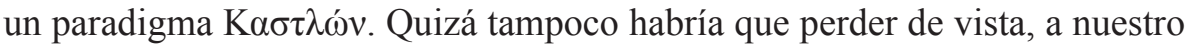
juicio, como apoyo secundario las variantes que aparecen en algunas ediciones de Estrabón y otros autores pertenecientes a un paradigma K $\alpha \sigma \tau \alpha \lambda \omega ́$, K $\alpha \sigma \tau \alpha \lambda \tilde{\omega} \nu$ S, que presentan una vocal anaptíctica muy griega, la $a$ (uid. en este sentido las referencias que aparecen en TIR J-30, s. u. Castulo). En cualquier caso, en kaśtilo se utilizó el silabograma ti y el fonemograma l para solventar la escritura de la secuencia -tl-. La razón que aducía Correa (p. 109), con gran prudencia, para esa solución era gráfica: el silabograma ti, al igual que ka, podrían haberse utilizado para indicar los fonemas $t$ y $k$, tal y como parecía indicar su uso en algunas ocasiones seguido por los fonemogramas i y a. Nosotros opinamos, sin embargo, que bien podría pensarse o también podría pensarse en razones gráfico-fonéticas o, por mejor decir, dependería de la pericia del escribiente para resolver gráficamente un grupo de muta cum liquida. En la solución adoptada podría intervenir desde la tradición escrituraria en la que se hallaba hasta el entorno fónico, en caso de no existir la primera o de ser muy débil. Desde este punto de vista, quizá haya que entender el mismo fenómeno en baitolo (A.8) y su forma latina Baetulo. En la leyenda ibérica, de zona nordoriental ahora, se utiliza otro procedimiento, el uso del silabograma con la misma vocal que sigue a la líquida. Es decir, que el topónimo "sonaría» originariamente más o menos [ba’ytlo].

2. De lo anterior se desprende que la líquida no sería geminada en origen, sino que se introduciría al adaptarlo al latín, sin que podamos determinar las causas. No parece esperable una forma *atllu.

La forma [atlūnkūm] se resolvió de una de las tres formas posibles gráficamente que hemos confirmado por ahora en celtibérico, el tipo konterbia: atulnkum. El tipo kolounioku habría sido *atulunkum; el kontebakom, *atunkum. 
Desde el punto de vista morfológico [atlūnkūm] es el genitivo del plural de un adjetivo *atlūnkos, atlūnka, atlünkom, derivado de un tema en nasal *atlūn- < *atlōn, con el ya mencionado sufijo individualizador con el grado alargado generalizado, más el sufijo $-k o$.

Creemos que una forma que presenta una estructura morfológica de derivación igual es la que aparece en una inscripción de la Cueva de la Griega (Segovia) en la que se lee Nem[e]do / Augusto / Aiunc[o] Ne? Medani (HEp 7, 1997, 690. Anteriormente $A E$ 1997, 894, en donde se indica que el principio de la línea 3 podría ser, según la foto, nunc? $=H E p 5$ 1995, 685). No está claro si se trata de un epíteto de la divinidad, pero tiene el aspecto de ser una forma de un paradigma celtibérico (o casi) *aiūnkos, -a, -om, con una vocal $-\bar{o}-$, procedente de un tema débil *aī̄n-, con la extensión analógica de la vocal del tema fuerte. Este tema conforma un antropónimo que está testimoniado en lengua y epigrafía celtibérica como aiu ([K.1.1, B-3] aiu berkantikum / abulos kentis); y en epigrafía y lengua latina, como Aio (CIL II 5790), posible nominativo, cuyo genitivo sería el también testimoniado Aionis (CIL II 5790). No se ha encontrado todavía el correspondiente genitivo celtibérico regularizado *aiunos.

La forma Aiunc [o] tiene el aspecto, a nuestro juicio, de ser fruto de una interferencia lingüística precisamente por la presencia de la $-u-$. La inscripción parece que comienza en latín, pues mantiene la $-\bar{o}$ final del dativo, pero cuando llega a Aiunc[o] utiliza la forma con - $u$ - en lugar de la esperada con -o-, a juzgar por el ginecónimo que debe estar relacionado con él y debe ser su adaptación latina, Aionca, que está testimoniado en Lara de los Infantes, Burgos (como dativo en ERLara 155: Ambatae Aioncae T[i]ti f.; 185: Ambatae Aioncae Lougei f.; y como nominativo, aunque con restitución de las dos primeras letras en ERLara 191: [Am]bata [Ai]onca [Cae?]niuetis f; vid. Vallejo 2005, p. 111). Sobre la constitución morfológica de la familia lingüística aiu/Aio, aiankum, Aionca, nos ocupamos con detenimiento en otro lugar (Jordán e. p.).

Si se nos pregunta qué interpretación preferimos para atulnkum, la A) [atulnkūm] o la B) [atlūnkūm], diremos que esta última. Sin embargo, como casi siempre, deberemos esperar más datos para confirmar el error del grabador o nuestro acierto o fallo para explicar la forma. Y en la espera el desasosiego crece, cuando se contempla la pieza CP-3, de la colección Pellicer, turiaz/nka (!) / kortika. La forma, la paleo-epigrafía y la morfología del adjetivo nos llevaron a catalogarla como sospechosa (Beltrán, Jordán y Simón 
2009). Sin embargo, nos encontramos ante el mismo error gráfico, $\mathbf{n}$ por $\mathbf{i}$, estando implicado de nuevo un tema en nasal, turiazu (A.51). ¿Casualidad?

\section{BIBLIOGRAFÍA}

Abascal, J. A. 1994: Los nombres personales en las inscripciones latinas de Hispania, Murcia.

Albertos, M. ${ }^{\text {a }}$ L. 1966: La onomástica personal primitiva de Hispania: Tarraconense y Bética, Salamanca.

Albertos, M. ${ }^{a}$ L. 1972: «Nuevos antropónimos hispánicos (2. ${ }^{a}$ serie)», Emerita 40, pp. 1-29 у 287-318.

Albertos, M. ${ }^{a}$ L. 1975: «Organizaciones suprafamiliares en la Hispania Antigua», Studia Archaeologica 37, pp. 5-66.

Albertos, M. ${ }^{a}$ L. 1977: «Correcciones a los trabajos sobre onomástica personal indígena de M. Palomar Lapesa y M. ${ }^{a}$ Lourdes Albertos Firmat», Emerita 45, pp. 33-54.

Almagro-Gorbea, M. 2003: Epigrafia prerromana, Madrid.

Almagro-Gorbea, M. et al. 2004: Prehistoria. Antigüedades Españolas I, Madrid.

Balbín, P. 2006: Hospitalidad y patronato en la Península Ibérica durante la Antigüedad, Salamanca.

Ballester, X. 2004: «Notas a epígrafes celtibéricas de colecciones particulares», PalHisp 4, pp. 265-282.

BB III: Beltrán, F., Hoz, J. de y Untermann, J., 1996: El tercer Bronce de Botorrita (Contrebia Belaisca), Zaragoza, 1996.

Beltrán, F. 2001: La hospitalidad celtibérica: una aproximación desde la epigrafía latina», PalHisp 1, pp. 35-62.

Beltrán, F., Jordán, C. y Simón, I. 2009: «Revisión y balance del corpus de téseras celtibéricas», en Beltrán, F. et al. (eds.), Acta Palaeohispanica X (Actas do X Colóquio sobre línguas e culturas paleo-hispânicas. Lisboa, 26-28 de Fevereiro de 2009), Zaragoza = PalHisp 9, pp. 625-668.

Correa, J. A. 1983: «Ibérico: caśt(i)lo, ibolc(a). Latín: Castulo, Obulco», Habis 14, pp. 107-113.

Curchin, L. 1994: «Juridical epigraphy and provincial administration in Central Spain», en González Fernández, J. (ed.), Roma y las Provincias. Realidad administrativa e ideología imperial, Madrid, pp. 87-102.

D’Encarnação, J. 1975: Divindades indígenas sob o domínio romano em Portugal, Lisboa.

ERLara $=$ Abásolo, J. A. 1974: Epigrafia romana de la región de Lara de los Infantes, Burgos. 
Hamp, E. 1989: «On Celtiberian Nasal Stems», ZCPh 43, pp. 188-189.

Heras Fernández, E. 1993: «Una inscripción funeraria procedente de Alcozar (Soria)», Ficheiro Epigráfico 44, n. ${ }^{\circ} 200$.

Hoz, J. de 1986: «La epigrafía celtibérica», en Actas de la Reunión sobre Epigrafía Hispánica de época romano-republicana, Zaragoza, pp. 41-102.

IRCP = D'Encarnação, J. 1984: Inscrições romanas do Conventus Pacensis. Subsídios para o estudo da romanização, 2 vols., Coimbra.

Jordán, C. 2003: «Acerca del ablativo que aparece en las téseras de hospitalidad celtibéricas», PalHisp 3, pp. 113-127.

Jordán, C. 2004a: «Chronica Epigraphica Celtiberica III», PalHisp 4, pp. 285323.

Jordán, C. 2004b: «Sobre la interpretación de los mensajes contenidos en las téseras de hospitalidad celtibéricas», ELEA 6, pp. 161-191.

Jordán, C. 2004c: Celtibérico, Zaragoza.

Jordán, C. 2007: «Estudios sobre el sistema dual de escritura en epigrafía no monetal celtibérica», PalHisp 7, pp. 101-142.

Jordán, C. 2008: «El Valle Medio del Ebro como zona de contacto lingüístico de las lenguas paleohispánicas», REL 38/1, pp. 5-32.

Jordán, C. e. p.: «La secuencia -anko- en la antroponimia celtibérica», Continental Celtic Word Formation: Onomastic Data, Salamanca.

Mesa, J. F. 1998: «Tres nuevos grafitos, dos fragmentos de inscripción y marcas sobre columna localizados en el Foro de Caesaraugusta», Caesaraugusta 74, pp. 197-206.

MLH IV = Untermann, J. 1997: Monumenta Linguarum Hispanicarum IV. Die tartessischen, keltiberischen und lusitanischen Inschriften, Wiesbaden.

Palomar, M. 1957: La onomástica personal pre-latina de la antigua Lusitania, Salamanca.

Ramírez, J. L. y Gijón, E. 1994: «Las inscripciones de la Necrópolis del Albarregas (Mérida) y su contexto arqueológico», Veleia 11, pp. 117-167.

Rubio, F. 2001: «Las formaciones secundarias en -ko- del celtibérico», en Villar, F. y Fernández, M. ${ }^{a}$ P. (eds.), Religión, Lengua y Cultura Prerromanas de Hispania. Actas del VIII Coloquio sobre Lenguas y Culturas Prerromanas de la Península Ibérica (Salamanca, 11-15 de mayo de 1999), Salamanca, pp. 581-594.

$T I R=$ Unión Académica Internacional 2001: Tabula Imperii Romani (Comité Español). Hoja J-30: Valencia, Madrid.

Tovar, A. 1983: «Una nueva pequeña tésera celtibérica», Emerita 51, pp. 1-3.

Untermann, J. 1990: «Comentarios sobre inscripciones celtibéricas 'menores'», en Villar, F. (ed.), Studia indogermanica et palaeohispanica in honorem A. Tovar et L. Michelena, Salamanca pp. 351-374. 
Vallejo, J. M. ${ }^{a}$ 2005: Antroponimia indígena de la Lusitania romana, Vitoria/Gasteiz.

Villar, F. 1995: Estudios de celtibérico y de toponimia prerromana, Salamanca.

Wodtko, D. 2000: Monumenta Linguarum Hispanicarum V.1. Wörterbuch der keltiberischen Inschriften, Wiesbaden.

Fecha de recepción de la primera versión del artículo: 03/05/2011

Fecha de aceptación: 18/01/2012

Fecha de recepción de la versión definitiva: 25/01/2012 